УДК 517.956.6

\title{
A Nonlocal Boundary Value Problem with Constant Coefficients for the Multidimensional Second Order Equation of Mixed Type of the Second Kind
}

\author{
Sirojiddin Z. Dzhamalov* \\ Institute of Mathematics \\ Uzbekistan Academy of Sciences \\ M. Ulugbek, Tashkent, 100170 \\ Uzbekistan
}

Received 06.11.2017, received in revised form 28.12.2017, accepted 15.02.2018

\begin{abstract}
Multidimensional second order equation of the mixed type of the second kind is considered in the paper. Unique solvability and smoothness of the solution of a nonlocal boundary value problem with constant coefficients in Sobolev spaces are proved under some conditions on coefficients.
\end{abstract}

Keywords: multidimensional equations, solvability, generalized solution.

\section{Introduction and formulation of the problem}

Let $\Omega=\prod_{i=1}^{n}\left(\alpha_{i}, \beta_{i}\right)$, be $n$-dimensional parallelepiped in the Euclidean space $\mathbb{R}^{n}$ of points $\left(x_{1}, \ldots, x_{n}\right), 0<\alpha_{i}<\beta_{i}<+\infty, \forall i=\overline{1, n}$.

In domain $Q=\Omega \times(0, T)$ we consider a second order differential equation

$$
L u=K(x, t) u_{t t}-\left(a_{i j}(x) u_{x_{i}}\right)_{x_{j}}+a(x, t) u_{t}+c(x, t) u=f(x, t) .
$$

Here and below repeating indexes mean summation from 1 to $n$. We assume that all functions below are real-valued and smooth enough.

Let $K(x, 0) \leqslant 0 \leqslant K(x, T)$ at $x \in \bar{\Omega}$. Then equation (1) is an equation of the mixed type of the second kind since function $K(x, t)$ can change sign in the domain $\bar{Q}[1-4]$.

\subsection{The nonlocal boundary value problem}

We are to find a generalized solution of equation (1) from Sobolev space $W_{2}^{\ell}(Q),(2 \leqslant \ell$ is a natural number) that satisfies nonlocal boundary conditions

$$
\begin{gathered}
\gamma \cdot u(x, 0)=u(x, T), \\
\left.\eta_{i} D_{x_{i}}^{p} u\right|_{x_{i}=\alpha_{i}}=\left.D_{x_{i}}^{p} u\right|_{x_{i}=\beta_{i}}
\end{gathered}
$$

when $p=0,1$, where $D_{x_{i}}^{p} u=\frac{\partial^{p} u}{\partial x_{i}^{p}}, D_{x_{i}}^{0} u=u, \gamma$ and $\eta_{i}, \forall i=\overline{1, n}$ are some constants which are not equal to zero. They will be defined below.

*siroj63@mail.ru

(C) Siberian Federal University. All rights reserved 
Nonlocal boundary value problems for the mixed type second order equation both first and second kinds were considered $[2,4-8,12,14,15]$. Nonlocal boundary value problems $(2),(3)$ for the mixed type equation of the first kind were studied for the first time by one of the authors of the paper [9].

Here equation (1) is considered in the case $K(x, 0) \leqslant 0 \leqslant K(x, T)$. Unique solvability and smoothness of the generalized solution of one nonlocal boundary value problem with constant coefficients (2), (3) in Sobolev spaces $W_{2}^{\ell}(Q)(2 \leqslant \ell \in \mathbb{N})$ are studied for the first time.

Let us assume that $a_{i j}(x)=a_{j i}(x) ; a_{i j}\left(\alpha_{k}\right)=a_{j i}\left(\beta_{k}\right), \forall k=\overline{1, n}$ end $\forall \xi \in \mathbb{R}^{n},|\xi|^{2}=\sum_{i=1}^{n} \xi_{i}^{2}$.

Let us also assume that one of the following conditions holds:

(a) $a_{i j} \xi_{i} \xi_{j} \geqslant a_{0}|\xi|^{2}$, where $a_{0}$ is const $>0$,

(b) $a_{i j} \xi_{i} \xi_{j} \leqslant a_{1}|\xi|^{2}$, where $a_{1}$ is const $<0$.

Further we assume that $\left|\eta_{i}\right| \geqslant 1,|\gamma|>1$ in the case of condition (a), $|\gamma|<1$ in the case of condition (b).

$W_{2}^{l}(Q)(2 \leqslant l$-natural number $)$ is the Sobolev space with the scalar product $(,)_{l}$ and the norm $\|\cdot\|_{l}, W_{2}^{0}(Q)=L_{2}(Q)$ is the space of square integrable functions.

Let $\nu=\left(\nu_{t}, \nu_{x_{1}}, \ldots, \nu_{x_{n}}\right)$ be a unit vector of an exterior normal to the boundary $\partial Q$, where $\nu_{t}=\cos (\nu, t), \nu_{x_{i}}=\cos \left(\nu, x_{i}\right), \forall i=\overline{1, n}$.

Further, the Young inequality is often used

$$
\forall u, v>0, \forall \sigma>0, p>1, \quad u \cdot v \leqslant \frac{\sigma^{p} u^{p}}{p}+\frac{v^{q}}{q \sigma^{q}}, \frac{1}{p}+\frac{1}{q}=1 .
$$

If $p=q=2$ then we come to the Cauchy inequality with $\sigma$ [10].

First, we consider the case $l=2$, that is, $u \in W_{2}^{2}(Q)$ and assume that coefficients of equation (1) are smooth enough functions.

\section{Uniqueness of the solution of the problem}

Theorem 2.1. Let us assume that above mentioned conditions on coefficients of equation (1) are fulfilled and $2 a-K_{t}+\lambda K \geqslant \delta_{1}>0, \lambda c-c_{t} \geqslant \delta_{2}>0$, where $\lambda=\frac{2}{T} \ln |\gamma|>0$ if $|\gamma|>1$ in the case of condition (a) and $\lambda=\frac{2}{T} \ln |\gamma|<0$ if $|\gamma|<1$ in the case of condition (b), $\left|\eta_{i}\right| \geqslant 1$, $\forall i=\overline{1, n}, c(x, 0) \leqslant c(x, T)$. If a generalized solution of problem (1)-(3) from the space $W_{2}^{2}(Q)$ exists for any function $f \in L_{2}(Q)$ then the solution is unique and the following inequality holds:

$$
\|u\|_{1} \leqslant m\|f\|_{0} .
$$

From this point on $m$ is positive constant.

Proof. Let us assume that a generalized solution of problem (1)-(3) exists in the space $W_{2}^{2}(Q)$. Taking into account conditions of Theorem 1 and the Cauchy inequality with $\sigma$ from problem (1)-(3), it is easy to obtain the following inequality

$$
\begin{aligned}
2 \int_{Q} L u & \exp \left(-\lambda t-\sum_{i=1}^{n} \mu_{i} x_{i}\right) \cdot u_{t} d x d t \geqslant \int_{Q} \exp \left(-\lambda t-\sum_{i=1}^{n} \mu_{i} x_{i}\right)\left\{\left(2 a-K_{t}+\lambda K\right) \cdot u_{t}^{2}+\right. \\
& \left.+\lambda a_{i j} u_{x_{i}} u_{x_{j}}+\left(\lambda c-c_{t}\right) \cdot u^{2}\right\} d x d t-\sigma \cdot\left\|u_{x}\right\|_{0}^{2}-\mu^{2} \sigma^{-1} \cdot\left\|u_{t}\right\|_{0}^{2}+ \\
& +\int_{\partial Q} \exp \left(-\lambda t-\sum_{i=1}^{n} \mu_{i} x_{i}\right)\left\{K u_{t}^{2} \nu_{t}-2 a_{i j} u_{x_{i}} u_{t} \nu_{x_{i}}+a_{i j} u_{x_{i}} u_{x_{j}} \nu_{t}+c \cdot u^{2} \nu_{t}\right\} d s,
\end{aligned}
$$


where $0 \leqslant \mu_{i}=\frac{2}{\theta_{i}} \ln \left|\eta_{i}\right|, 0<\theta_{i}=\left(\beta_{i}-\alpha_{i}\right), \sigma$ and $\sigma^{-1}$ are coefficients of the Cauchy inequality with $\sigma$. Conditions of Theorem 1 provide non-negativity of the integral over the domain $Q$ and on the boundary $\partial Q$. Because $u \in W_{2}^{2}(Q)$ satisfies boundary conditions (2), (3) and $\gamma^{2}=e^{-\lambda \cdot T}$, $\eta_{i}^{2}=e^{\mu_{i} \cdot \theta_{i}}$ then

$$
\begin{gathered}
\int_{\partial Q} \exp \left(-\lambda t-\sum_{i=1}^{n} \mu_{i} x_{i}\right)\left\{K u_{t}^{2} \nu_{t}-2 a_{i j} u_{x_{i}} u_{t} \nu_{x_{i}}+a_{i j} u_{x_{i}} u_{x_{j}} \nu_{t}+c u^{2} \nu_{t}\right\} d s= \\
=\int_{\alpha_{i}}^{\beta_{i}} \exp \left(-\sum_{i=1}^{n} \mu_{i} x_{i}\right)\left\{\left[K(x, T) e^{-\lambda T} \gamma^{2}-K(x, 0)\right] u_{t}^{2}(x, 0)+\right. \\
\left.\quad+\left[e^{-\lambda t} \gamma^{2}-1\right] u_{x_{i}}^{2}(x, 0)+\left[c(x, T) e^{-\lambda T} \gamma^{2}-c(x, 0)\right] u^{2}(x, 0)\right\} d x- \\
\quad-2\left[\exp \left(-\mu_{i} \beta_{i}\right) \eta_{i}^{2}-\exp \left(-\mu_{i} \alpha_{i}\right)\right] \int_{0}^{T} \exp (-\lambda t) u_{x_{i}}\left(-\alpha_{i}, t\right) u_{t}\left(\alpha_{i}, t\right) d t \geqslant \\
\geqslant \int_{\alpha_{i}}^{\beta_{i}} \exp \left(-\sum_{i=1}^{n} \mu_{i} x_{i}\right)\left\{\left[K(x, T) e^{-\lambda T} \gamma^{2}-K(x, 0)\right] u_{t}^{2}(x, 0)+\right. \\
\left.+\left[c(x, T) e^{-\lambda t} \gamma^{2}-c(x, 0)\right] u^{2}(x, 0)\right\} d x \geqslant 0 .
\end{gathered}
$$

Omitting positive boundary integrals, we obtain from (5) the following inequality

$$
\begin{array}{r}
2 \int_{Q} L u \cdot \exp \left(-\lambda t-\sum_{i=1}^{n} \mu_{i} x_{i}\right) \cdot u_{t} d x d t \geqslant \int_{Q} \exp \left(-\lambda t-\sum_{i=1}^{n} \mu_{i} x_{i}\right)\left\{\left(2 a-K_{t}+\lambda K\right) \cdot u_{t}^{2}+\right. \\
\left.+\lambda a_{\tau} u_{x_{i}}^{2}+\left(\lambda c-c_{t}\right) \cdot u^{2}\right\} d x d t-\sigma\left\|u_{x_{i}}\right\|_{0}^{2}-\mu^{2} \cdot \sigma^{-1} \cdot\left\|u_{t}\right\|_{0}^{2}
\end{array}
$$

where $a_{\tau}=a_{0}$ in the case of condition $(a), a_{\tau}=a_{1}$ in the case of condition (b). Setting coefficients $\lambda a_{\tau}-\sigma \geqslant \lambda_{0}>0, \delta_{1}-\mu^{2} \sigma^{-1}>\delta_{0}>0$, we obtain from inequality (6) the first a priori estimate

$$
\|u\|_{1} \leqslant m\|f\|_{0} .
$$

Uniqueness of the generalized solution of problem $(1)-(3)$ in $W_{2}^{2}(Q)$ follows from this estimate.

\section{The equations of composite type}

To prove the existence of the solution of problem $(1)-(3)$ in $W_{2}^{2}(Q)$ we use the method of " $\varepsilon$-regularisation" together with Galerkin method $[1,3,8,13]$.

Let us consider a nonlocal problem for composite type equation

$$
\begin{gathered}
L_{\varepsilon} u_{\varepsilon}=-\varepsilon \frac{\partial}{\partial t} \Delta u_{\varepsilon}+L u_{\varepsilon}=f(x, t), \\
\left.\gamma D_{t}^{q} u_{\varepsilon}\right|_{t=0}=\left.D_{t}^{q} u_{\varepsilon}\right|_{t=T}, \quad q=0,1,2, \\
\left.\eta_{i} D_{x_{i}}^{p} u_{\varepsilon}\right|_{x_{i}=\alpha_{i}}=\left.D_{x_{i}}^{p} u_{\varepsilon}\right|_{x_{i}=\beta_{i}}, \quad p=0,1,
\end{gathered}
$$

where $\Delta u=\frac{\partial^{2} u}{\partial t^{2}}+\sum_{i=1}^{n} \frac{\partial^{2} u}{\partial x_{i}^{2}}$ is the Laplace operator, $D_{x_{i}}^{p} u=\frac{\partial^{p} u}{\partial x_{i}^{p}}, \quad D_{x_{i}}^{0} u=u, \quad p=0,1$, $D_{t}^{q} u=\frac{\partial^{q} u}{\partial t^{q}}, q=1,2 ; \quad D_{t}^{0} u=u, \varepsilon$ is a small enough positive number, $\eta_{i}, \gamma=$ const $\neq 0$, such that $|\gamma|>1$ in the case of condition $(a),|\gamma|<1$ in the case of condition $(b),\left|\eta_{i}\right| \geqslant 1, \forall i=\overline{1, n}$. 
In what follows we use composite type equation (7) as the $\varepsilon$-regularization equation for equation (1) $[1,8]$.

Let us denote a class of functions such that $u_{\varepsilon}(x, t) \in W_{2}^{2}(Q)$ and $\frac{\partial \Delta u_{\varepsilon}}{\partial t} \in L_{2}(Q)$ satisfying conditions (8),(9) by $W$.

Definition. Function $u_{\varepsilon}(x, t) \in W$ satisfying equation (7) is denoted the regular solution of problem (7)-(9).

Theorem 3.1. Let us assume that above mentioned coefficient conditions for equation (1) are fulfilled and $2 a-\left|K_{t}\right|+\lambda \geqslant \delta_{1}>0, \lambda c-c_{t} \geqslant \delta_{2}>0$, where $\lambda=\frac{2}{T} \ln |\gamma|>0$ if $|\gamma|>1$ in the case of condition $(a)$ and $\lambda=\frac{2}{T} \ln |\gamma|<0$ if $|\gamma|<1$ in the case of condition $(b),\left|\eta_{i}\right| \geqslant 1$, $c(x, 0)=c(x, T), a(x, 0)=a(x, T), a\left(\alpha_{i}, t\right)=a\left(\beta_{i}, t\right), K\left(\alpha_{i}, t\right)=K\left(\beta_{i}, t\right), \forall i=\overline{1, n}$. Then for any function $f, f_{t} \in L_{2}(Q)$, such that $\gamma \cdot f(x, 0)=f(x, T)$ there is a unique regular solution of problem (7)-(9), and the following inequalities are true:

$$
\begin{aligned}
& \text { I) } \quad \varepsilon\left(\left\|u_{\varepsilon t t}\right\|_{0}^{2}+\left\|u_{\varepsilon t x}\right\|_{0}^{2}\right)+\left\|u_{\varepsilon}\right\|_{1}^{2} \leqslant m\|f\|_{0}^{2}, \\
& I I) \quad \varepsilon\left\|\frac{\partial \Delta u_{\varepsilon}}{\partial t}\right\|_{0}^{2}+\left\|u_{\varepsilon}\right\|_{2}^{2} \leqslant m\left[\|f\|_{0}^{2}+\left\|f_{t}\right\|_{0}^{2}\right] .
\end{aligned}
$$

Proof. The proof of Theorem 2 is carried out using Galerkin method with special basis functions. $[8,10]$.

\subsection{Proof of the first a priori estimate I)} lem

Consider the following spectral problems. Let $\phi_{j}(x, t)$ be eigenfunction of the following prob-

$$
\begin{gathered}
\Delta \phi_{j}=\frac{\partial^{2} \phi_{j}}{\partial^{2} t}+\frac{\partial^{2} \phi_{j}}{\partial^{2} x}=-\nu_{j}^{2} \phi_{j}, \\
\left.D_{t}^{p} \phi_{j}\right|_{t=0}=\left.D_{t}^{p} \phi_{j}\right|_{t=T}, \quad p=0,1, \\
\left.D_{x}^{p} \phi_{j}\right|_{x=0}=\left.D_{x}^{p} \phi_{j}\right|_{x=\ell} .
\end{gathered}
$$

It follows from the general theory of linear self-adjoint elliptic operators that all $\left\{\phi_{j}(x, t)\right\}$ are eigenfunctions of problem (10)-(12). They form fundamental system in $W_{2}^{2}(Q)$, and they are orthonormal in $L_{2}(Q)[10,11]$. Then we construct the solution of an auxiliary problem using these functions:

$$
\begin{gathered}
\exp \left[\frac{-1}{2}\left(\lambda t+\sum_{i=1}^{n} \mu_{i} x_{i}\right)\right] \omega_{j t}=\phi_{j} \\
\gamma \cdot \omega_{j}(x, 0)=\omega_{j}(x, T)
\end{gathered}
$$

where, $\gamma=$ const $\neq 0$, such that $|\gamma|>1$ in the case of condition $(a),|\gamma|<1$ in the case of condition $(b), 0 \leqslant \mu_{i}=\frac{2}{\theta_{i}} \ln \left|\eta_{i}\right|,\left|\eta_{i}\right| \geqslant 1, \forall i=\overline{1, n}$. Obviously, problem (13), (14) is uniquely solvable and its solution has the from

$$
\ell^{-1} \phi_{j}=\omega_{j}=\exp \left(\frac{\sum_{i=1}^{n} \mu_{i} \cdot x_{i}}{2}\right) \cdot\left[\int_{0}^{t} \exp \left(\frac{\lambda \tau}{2}\right) \phi_{j} d \tau+\frac{1}{\gamma-1} \int_{0}^{T} \exp \left(\frac{\lambda t}{2}\right) \phi_{j} d t\right] .
$$


It is clear that functions $\omega_{j}(x, t)$ are linearly independent. Indeed, if $\sum_{j=1}^{N} c_{j} \omega_{j}=0$ for some set of functions $\omega_{1}, \omega_{2}, \ldots, \omega_{N}$ then acting on this sum by the operator $\ell$, we have $\sum_{j=1}^{N} c_{j} \ell \omega_{j}=$ $=\sum_{j=1}^{N} c_{j} \phi_{j}=0$. Then we obtain that $c_{j}=0$ for any $j=\overline{1, N}$. It follows from the construction of function $\phi_{j}(x, t)$ that functions $\omega_{j}(x, t)$ satisfy the following conditions

$$
\begin{gathered}
\left.\gamma D_{t}^{q} \omega_{i}\right|_{t=0}=\left.D_{t}^{q} \omega_{i}\right|_{t=T}, \quad q=0,1,2 \\
\left.\eta_{i} D_{x_{i}}^{p} \omega_{i}\right|_{x_{i}=\alpha_{i}}=\left.D_{x_{i}}^{p} \omega_{i}\right|_{x_{i}=\beta_{i}}, \quad p=0,1 .
\end{gathered}
$$

We take the approximate solution of (7)-(9) in the from $w=u_{\varepsilon}^{N}=\sum_{j=1}^{N} c_{j} \omega_{j}$ where coefficients $c_{j}$ are defined for any $j=\overline{1, N}$ as solutions of the linear algebraic system

$$
\int_{Q} L_{\varepsilon} u_{\varepsilon}^{N} \cdot e^{\frac{-\left(\lambda \cdot t+\sum_{i=1}^{n} \mu_{i} \cdot x_{i}\right)}{2}} \phi_{j} d x d t=\int_{Q} f \cdot e^{\frac{-\left(\lambda \cdot t+\sum_{i=1}^{n} \mu_{i} \cdot x_{i}\right)}{2}} \phi_{j} d x d t
$$

We prove the unique solvability of algebraic system (18). Multiplying every equation of (18) by $2 c_{j}$ and summing up with respect to $j$ from 1 to $N$ and taking into account (12), (13), (18), we obtain

$$
\int_{Q} L_{\varepsilon} w \cdot e^{-\left(\lambda t+\sum_{i=1}^{n} \mu_{i} x_{i}\right)} \cdot w_{t} d x d t=\int_{Q} f \cdot e^{-\left(\lambda t+\sum_{i=1}^{n} \mu_{i} x_{i}\right)} \cdot w_{t} d x d t
$$

Upon integrating identity (19), by virtue of theorem 2 we obtain for the approximate solution of problem (7)-(9) the estimates I), i.e.

$$
\varepsilon\left(\left\|u_{\varepsilon t t}^{N}\right\|_{0}^{2}+\left\|u_{\varepsilon t x}^{N}\right\|_{0}^{2}\right)+\left\|u_{\varepsilon}^{N}\right\|_{1}^{2} \leqslant m\|f\|_{0}^{2} .
$$

This implies the solvability of algebraic system (18). In particular, from estimate (20) we obtain a weak solution of problem (7)-(9) $[3,10]$.

\subsection{Proof of the second a priori estimate II.)}

Taking into account problem (10)-(14), from identity (18) we obtain

$$
-\frac{1}{\nu_{j}^{2}} \int_{Q} L_{\varepsilon} w e^{\frac{-\left(\lambda \cdot t+\sum_{i=1}^{n} \mu_{i} \cdot x_{i}\right)}{2}} \Delta \ell \omega_{j} d x d t=-\frac{1}{\nu_{j}^{2}} \int_{Q} f e^{\frac{-\left(\lambda \cdot t+\sum_{i=1}^{n} \mu_{i} \cdot x_{i}\right)}{2}} \Delta \ell \omega_{j} d x d t,
$$

where,

$\Delta \ell \omega_{j}=\exp \left[\frac{-\left(\lambda t+\sum_{i=1}^{n} \mu_{i} x_{i}\right)}{2}\right]\left(\Delta \omega_{j_{t}}-\lambda \omega_{j_{t t}}-\mu_{j} \omega_{j_{x x}}+\frac{\lambda^{2}+\mu_{j}^{2}}{4} \omega_{j_{t}}\right), \Delta \omega_{j}=\omega_{j_{t t}}+\omega_{j_{x x}}$.

Multiplying each equation of (21) by $2 \nu_{j}^{2} c_{j}$ and summing up with respect to $j$ from 1 to $N$ and considering (15), (16), (21), we have the following identity

$$
-2 \int_{Q} L_{\varepsilon} w \cdot e^{\frac{-\left(\lambda \cdot t+\sum_{i=1}^{n} \mu_{i} x_{i}\right)}{2}} \cdot \Delta \ell w d x d t=-2 \int_{Q} f \cdot e^{\frac{-\left(\lambda \cdot t+\sum_{i=1}^{n} \mu_{i} x_{i}\right)}{2}} \cdot \Delta \ell w d x d t .
$$


Integrating (22) and taking into account conditions of Theorem 2.1 and boundary conditions (15), (16), we obtain the following inequality

$$
\begin{gathered}
m \cdot\left[\left\|f_{t}\right\|_{0}^{2}+\|f\|_{0}^{2}\right] \geqslant \varepsilon\left\|\frac{\partial \Delta w}{\partial t}\right\|_{0}^{2}+\int_{Q} e^{-\left(\lambda \cdot t+\sum_{i=1}^{n} \mu_{i} x_{i}\right)}\left\{\left(2 \alpha-\left|K_{t}\right|+\lambda K\right) w_{t t}^{2}+\right. \\
\left.+\left(2 \alpha-\left|K_{t}\right|+\lambda K\right) w_{t x_{i}}^{2}+\lambda w_{x_{i} x_{i}}^{2}+\lambda w_{t x_{i}}^{2}\right\} d x d t+\int_{\partial Q} e^{-\left(\lambda \cdot t+\sum_{i=1}^{n} \mu_{i} x_{i}\right)}\left[\left(K w_{t t}^{2}-2 \alpha w_{t} w_{t t}+\right.\right. \\
+w_{x_{i} x_{i}}^{2}+2 w_{x_{i} x_{i}} w_{t t}-w_{x_{i} t}^{2}+K w_{x_{i} t}^{2}+2 c w\left(w_{t t}+w_{x_{i} x_{i}}\right) \nu_{t}+ \\
\left.+\left(2 K w_{t t} w_{x_{i} t}-2 w_{t t} w_{x_{i} t}+2 \alpha w_{t} w_{x_{i} t}\right) \nu_{x_{i}}\right] d s-\sigma\left(\left\|w_{x x}\right\|_{0}^{2}+\left\|w_{x t}\right\|_{0}^{2}\right)- \\
-\mu^{2} \sigma^{-1}\left\|u_{t t}\right\|_{0}^{2}-m\left(\|f\|_{0}^{2}\right)=\sum_{i=1}^{2} J_{i}, \quad(23)
\end{gathered}
$$

where, $J_{1}$ is the integral over the domain, $J_{2}$ is the integral over the boundary.

Taking into account conditions of Theorem 2.1 and boundary conditions (14), (15), we obtain for coefficients $\lambda-\sigma \geqslant \lambda_{0}>0, \delta_{1}-\mu^{2} \sigma^{-1}>\delta_{0}>0$ that $J_{1}>0$ and $J_{2} \geqslant 0$. Now we have from inequality (23) the second estimate

$$
\varepsilon \cdot\left\|\frac{\partial}{\partial t} \Delta u_{\varepsilon}^{N}\right\|_{0}^{2}+\left\|u_{\varepsilon}^{N}\right\|_{2}^{2} \leqslant m \cdot\left[\|f\|_{0}^{2}+\left\|f_{t}\right\|_{0}^{2}\right] .
$$

Hence, from the well-known theorem on weak compactness [10] the obtained estimations (20), (24) allow one to take the limit $N \rightarrow \infty$ and to conclude that a subsequence $\left\{u_{\varepsilon}^{N_{k}}\right\}$ converges in $L_{2}(Q)$ together with the first and the second order derivatives to the unique regular solution $u_{\varepsilon}(x, t)$ of problem (7)-(9) with the properties specified in Theorem $2.1[3,6,8,10]$.

By virtue of (24) the following inequality holds for $u_{\varepsilon}(x, t)$

$$
\varepsilon\left\|\frac{\partial}{\partial t} \Delta u_{\varepsilon}\right\|_{0}^{2}+\left\|u_{\varepsilon}\right\|_{2}^{2} \leqslant m \quad\left[\|f\|_{0}^{2}+\left\|f_{t}\right\|_{0}^{2}\right] .
$$

Theorem 2.1 is proved.

\section{Existence of solution for the problem}

\subsection{The method of " $\varepsilon$-regularization"}

Now by means of the method of " $\varepsilon$-regularization" we prove solvability of problem (1)-(3).

Theorem 4.1. Let us assume that all conditions of theorem 2.1 are satisfied. Then the generalized solution of problem (1)(3) in space $W_{2}^{2}(Q)$ exists and it is unique

Proof. The uniqueness of the solution of problem $(1)-(3)$ in $W_{2}^{2}(Q)$ is proved in Theorem 1.1. Now we prove existence of the generalized solution of problem $(1)-(3)$ in $W_{2}^{2}(Q)$. For this purpose, we consider equation (7) in the domain $Q$ with nonlocal boundary conditions (8), (9) at $\varepsilon>0$. Because all conditions of Theorem 2.1 are fulfilled then there exists unique regular solution of problem (7)-(9) at $\varepsilon>0$, and estimates I),II) are true for it.

It follows from the well-known theorem on weak compactness [10] that it is possible to take from the set of functions $\left\{u_{\varepsilon}\right\}, \varepsilon>0$ weakly converging sub sequence of functions in $W$ such that $\left\{u_{\varepsilon_{i}}\right\} \rightarrow u$ at $\varepsilon_{i} \rightarrow 0$. Let us show that limit function $u(x, t)$ satisfies the equation $L u=f(1)$. 
Indeed, as sequence $\left\{u_{\varepsilon_{i}}\right\}$ converges weakly in $W_{2}^{2}(Q)$, sequence $\frac{\partial \Delta u_{\varepsilon}}{\partial t},(\varepsilon>0)$ is uniformly bounded in $L_{2}(Q)$, and operator $L$ is linear, then we have

$$
L u-f=L u-L u_{\varepsilon_{i}}+\varepsilon_{i} \cdot \frac{\partial \Delta u_{\varepsilon_{i}}}{\partial t}=L\left(u-u_{\varepsilon_{i}}\right)+\varepsilon_{i} \cdot \frac{\partial \Delta u_{\varepsilon_{i}}}{\partial t} .
$$

Taking the limit $\varepsilon_{i} \rightarrow 0$, we obtain from (26) the unique solution of problem (1)-(3) in $W_{2}^{2}(Q)$ $[1,6,8]$.

Theorem 3.1 is proved.

\section{Smoothness of solution for the problem}

Now we prove a more general case $l \geqslant 3$. Further we assume that coefficients of equation (1) are infinitely differentiated in the closed domain $\bar{Q}$.

Theorem 5.1. Let us assume that conditions of Theorem 3.1 are fulfilled and

$$
\begin{aligned}
& 2\left(\alpha+p K_{t}\right)-\left|K_{t}\right|+\lambda K \geqslant \delta>0, \\
& \left.D_{t}^{m} K\right|_{t=0}=\left.D_{t}^{m} K\right|_{t=T},\left.\quad D_{t}^{m} a\right|_{t=0}=\left.D_{t}^{m} a\right|_{t=T},\left.\quad D_{t}^{m} c\right|_{t=0}=\left.D_{t}^{m} c\right|_{t=T} .
\end{aligned}
$$

Then for any function $f(x, t)$ such that $f \in W_{2}^{p}(Q), D_{t}^{p+1} f \in L_{2}(Q),\left.\gamma D_{t}^{m} f\right|_{t=0}=\left.D_{t}^{m} f\right|_{t=T}$ where $m=0,1,2,3, \ldots, p$ there exists unique generalized solution of problem (1)-(3) in the space $W_{2}^{p+2}(Q)$, where $p=1,2,3, \ldots$.

Proof. It follows from smoothness of the solution of problem (10)-(14) that the approximate solution of problem (7)-(9) satisfies conditions $w=u_{\varepsilon}^{N} \in C^{\infty}(\bar{Q})$;

$$
\begin{aligned}
& \left.\gamma D_{t}^{q} w\right|_{t=0}=\left.D_{t}^{q} w\right|_{t=T}, \quad q=0,1,2, \ldots, \\
& \left.\eta_{i} D_{x_{i}}^{p} w\right|_{x_{i}=-\alpha_{i}}=\left.D_{x_{i}}^{p} w\right|_{x_{i}=\beta_{i}}, \quad p=0,1 .
\end{aligned}
$$

Taking into account conditions of Theorem 2.1 at $\varepsilon>0$, nonlocal conditions at $t=0, t=T$ and equality

$$
\left.\left(e^{-\frac{\lambda t}{2}} \cdot L_{\varepsilon} u_{\varepsilon}\right)\right|_{t=0} ^{t=T}=\left.\left(-\varepsilon \cdot e^{\frac{-\lambda t}{2}} \cdot \frac{\partial \Delta u_{\varepsilon}}{\partial t}+e^{\frac{-\lambda t}{2}} \cdot L u_{\varepsilon}\right)\right|_{t=0} ^{t=T}=\left.\left(e^{\frac{-\lambda t}{2}} \cdot f(x, t)\right)\right|_{t=0} ^{t=T},
$$

we obtain

$$
\left\|\gamma \cdot u_{\varepsilon t t t}(x, 0)-u_{\varepsilon t t t}(x, T)\right\|_{0} \leqslant \text { const. }
$$

Hence, function $v_{\varepsilon}(x, t)=u_{\varepsilon} t(x, t)$ belongs to $W$ and satisfies the following equation

$$
P_{\varepsilon} v_{\varepsilon}=L_{\varepsilon} v_{\varepsilon}=f_{t}-a_{t} u_{\varepsilon}-c_{t} u_{\varepsilon}=F_{\varepsilon} .
$$

It follows from theorem 2.1 that the set of functions $\left\{F_{\varepsilon}\right\}$ is uniformly bounded in the space $L_{2}(Q)$, i.e.

$$
\left\|F_{\varepsilon}\right\|_{0} \leqslant m\left[\|f\|_{0}^{2}+\left\|f_{t}\right\|_{0}^{2}\right] .
$$

Further, it can be easily obtained from conditions of Theorem 3.1 that coefficients of the operators $P_{\varepsilon}(\varepsilon>0)$ satisfy conditions of Theorem 4.1. Then on the basis of estimates I), II) for function $\left\{v_{\varepsilon}\right\}$ we obtain similar estimates

$$
\varepsilon\left(\left\|v_{\varepsilon t t}\right\|_{0}^{2}+\left\|v_{\varepsilon t x}\right\|_{1}^{2}\right)+\left\|v_{\varepsilon}\right\|_{1}^{2} \leqslant m\left(\|f\|_{0}^{2}+\left\|f_{t}\right\|_{0}^{2}\right),
$$




$$
\varepsilon\left\|\frac{\partial \Delta v_{\varepsilon}}{\partial t}\right\|_{0}^{2}+\left\|v_{\varepsilon}\right\|_{2}^{2} \leqslant m\left[\|f\|_{1}^{2}+\left\|f_{t t}\right\|_{0}^{2}\right] .
$$

Function $\left\{u_{\varepsilon}\right\}$ satisfies parabolic equation with conditions (2), (3)

$$
\Pi u_{\varepsilon}=u_{\varepsilon t}-\sum_{i, j=1}^{n}\left(a_{i j} u_{\varepsilon x_{i}}\right)_{x_{j}}=f+\varepsilon \frac{\partial \Delta u_{\varepsilon}}{\partial t}-K(x, t) u_{\varepsilon t t}-(a-1) u_{\varepsilon t}-c u_{\varepsilon}=\Phi_{\varepsilon},
$$

here $\Phi_{\varepsilon} \in L_{2}(Q)$. Set of functions $\left\{\Phi_{\varepsilon}\right\}$ is uniformly bounded in $W_{2}^{2}(Q)$, i.e.

$$
\left\|\Phi_{\varepsilon}\right\|_{0}^{2} \leqslant m\left[\|f\|_{1}^{2}+\left\|f_{t t}\right\|_{0}^{2}\right] \leqslant m\|f\|_{2}^{2} .
$$

On the basis of a priory estimates for parabolic equations [1], [10] and inequality (31) we obtain

$$
\left\|u_{\varepsilon}\right\|_{3}^{2} \leqslant m\|f\|_{2}^{2} .
$$

Further, one can prove in a similar way that $\left\|u_{\varepsilon}\right\|_{p+2}^{2} \leqslant m\|f\|_{p+1}^{2}$, where $p=2,3, \ldots$.

Remark. In the formulation of problem (1)-(3) the sign at the quadratic form does not play an essential role. However, in the case

(a) $a_{i j}(x) \xi_{i} \xi_{j} \geqslant a_{0}|\xi|^{2} ; a_{i j}=a_{j i}$, where $a_{0}=$ const $>0, x \in \Omega, \xi \in \mathbb{R}^{n}$

the class of equations (1) includes parabolic equations and in the case

(b) $a_{i j}(x) \xi_{i} \xi_{j} \leqslant a_{1}|\xi|^{2} ; a_{i j}=a_{j i}$, where $a_{1}=$ const $<0, x \in \Omega$

the class of equations (1) includes inverse parabolic equations. Nevertheless, similar results are obtained only with the change in the value of $\gamma$ for problem (1)-(3) in the case of conditions (a) and (b).

Therefore, the following question arises: whether or not restrictions on $\gamma$ are essential? In this connection we consider the following examples.

Examples. In the rectangle $Q=(0, \ell) \times(0, T)$ we consider the following problem

$$
\begin{gathered}
\Pi_{1} u=u_{t}-u_{x x}=0, \\
\gamma u(x, 0)=u(x, T), \\
u(0, t)=u(\ell, t)=0 .
\end{gathered}
$$

Solving problem (32)-(34) by the Fourier method, we find $\gamma_{k}=\exp \left(-\lambda_{k} T\right)<1, \lambda_{k}=\frac{2 \pi k}{\ell}$, $k=0,1,2, \ldots$. It is easy to verify that all conditions of Theorem 1 are fulfilled but functions $u_{k}=C_{k} e^{-\lambda_{k} t} \sin \lambda_{k} x$ (where $C_{k}$ are arbitrary constants) are nontrivial solutions of this boundary value problem.

In the same way, we consider the following problem

$$
\begin{gathered}
\Pi_{2} u=u_{t}+u_{x x}=0, \\
\gamma u(x, 0)=u(x, T), \\
u(0, t)=u(\ell, t)=0 .
\end{gathered}
$$

Solving problem (35)-(37) by the Fourier method, we find that functions $u_{k}=C_{k} e^{\lambda_{k} t} \sin \lambda_{k} x$ with any $C_{k}$ are nontrivial solutions of this boundary value problem. In this case $\gamma_{k}=\exp \left(\lambda_{k} T\right)>1$. 
Hence, we see that restrictions on $\gamma$ for both conditions (a) and (b) are essential. If these conditions are not satisfied then we do not have the uniqueness of the problem as shown above.

The author would like to thank prof. R. Ashurov and reviewer for useful comments and suggestions.

\section{References}

[1] V.N.Vragov, Boundary problems for non-classical equations of mathematical physics, Novosibirsk, 1983 (in Russian).

[2] M.G.Karatopraklieva, A nonlocal boundary-value problem for an equation of mixed type, Differents. Uravn., 27(1991), no. 1, 68-79 (in Russian).

[3] A.G.Kuzmin, Non-classical mixed type equations and their applications to the gas dynamics, Leningrad, 1990 (in Russian).

[4] S.N.Glazatov, Nonlocal boundary problems for mixed type equations in a rectangle, Siberian Math. J., 26(1985), no. 6, 162-164.

[5] N.A.Alimov, On a nonlocal boundary value problem for a non-classical equation. The theory and methods for solving ill-posed problems and their applications, Novosibirsk, 1983, 237 239 (in Russian).

[6] S.Z.Dzhamalov, On a nonlocal boundary value problem for a second order equation of mixed type of the second kind, Uzbek. Math. J., (2014), no. 1, 5-14 (in Russian).

[7] A.N.Terekhov, Nonlocal boundary problems for equations of variable type, Non-classical equations of mathematical physics, Novosibirsk, 1985, 148-158 (in Russian).

[8] S.Z.Djamalov, On the correctness of a nonlocal problem for the second order mixed type equations of the second kind in a rectangle, IIUM journal, 17(2016), no. 2, 95-104.

[9] S.Z.Dzhamalov, On nonlocal boundary value problem with constant coefficients for the equation of Trikomi, Uzbek. Mat. Zh., (2016), no. 2, 51-60 (in Russian).

[10] O.A.Ladyjenskaya, Boundary problems of mathematical physics, Moscow, 1973 (in Russian).

[11] Yu.M.Berezansky, Expansion in eigenfunctions of selfadjoint operators, Kyev, 1965 (in Russian).

[12] T.S.Kalmenov, About a floor to a periodic problem for the multidimensional equation mixed type, Differens. Uravn., 14(1978), no. 3, 546-548 (in Russian).

[13] A.I.Kozhanov, Boundary problems for equations of mathematical physics of odd order, Novosibirsk, 1990 (in Russian).

[14] K.B.Sabitov, Dirichlet problem for mixed type equations in rectangular domain, Dokl. Math. $R A N, \mathbf{7 5}(2007)$, no. 2, 193-196.

[15] B.N.Tsibikov, About a correctness of a periodic problem for the multidimensional equation mixed type, Non-classical equations of mathematical physics, 1986, 201-206 (in Russian). 
Об одной нелокальной краевой задаче с постоянным коэффициентом для многомерного уравнения смешанного типа второго рода, второго порядка

Сирожиддин 3. Джамалов

Институт математики

Академия наук Республики Узбекистан М. Улугбека, Ташкент, 100170

Узбекистан

$\bar{B}$ данной работе при выполнении некоторых условий на коэббициентъ многомерного уравнения смешанного типа второго рода в пространстве доказываются однозначная разрешимость и гладкость решения одной нелокальной краевой задачи с постоянным коэфбициентом в пространствах С.Л.Соболева.

Ключевые слова: многомерные уравнения, разрешимость, обобщенное решение. 